\title{
Arterite de Takayasu diagnosticada durante o puerpério em mulher com doença hipertensiva gestacional: relato de caso
}

\author{
Takayasu Arteritis diagnosed during puerperium in a woman with \\ pregnancy-induced hypertension: Case report
}

\begin{abstract}
Marta R. Hentschke1 ${ }^{1}$, Samanta Schneider², Fernando Sontag' ${ }^{3}$, Vicente B. Brentano ${ }^{3}$, Jeziel Basso ${ }^{4}$, Bartira E. Pinheiro da Costa ${ }^{5}$, Carlos E. Poli-de-Figueiredo ${ }^{6}$, Giovani Gadonski

${ }^{1}$ Médica. Doutora em Medicina pelo Programa de Pós-Graduação em Medicina e Ciências da Saúde (PPGMCS) da Pontifícia Universidade Católica do Rio Grande do Sul (PUCRS), Porto Alegre, RS; ${ }^{2}$ Médica graduada pela Faculdade de Medicina da PUCRS, Porto Alegre, RS; ${ }^{3}$ Acadêmicos da Faculdade de Medicina da PUCRS, Porto Alegre, RS; ${ }^{4}$ Médico graduado pela Faculdade de Medicina da PUCRS, Porto Alegre, RS; ${ }^{5}$ Doutora em Ciências pela Universidade Federal do Rio Grande do Sul (UFRGS), Professora Adjunta do Departamento de Medicina Interna da Faculdade de Medicina da PUCRS e do PPGMCS da PUCRS, Porto Alegre, RS; ${ }^{6}$ Médico Nefrologista, Doutor pela University of Oxford, Inglaterra, Professor Titular do Departamento de Medicina Interna da Faculdade de Medicina da PUCRS e do PPGMCS da PUCRS, ${ }^{7}$ Médico Nefrologista, Doutor em Medicina pelo PPGMCS da PUCRS, Porto Alegre, RS.
\end{abstract}

Estudo realizado com apoio da Fundação de Amparo à Pesquisa do Rio Grande do Sul (FAPERGS), do Conselho Nacional de Desenvolvimento Cientifico e Tecnológico (CNPq) e da Coordenação de Aperfeiçoamento de Pessoal de Nível Superior (CAPES).

\section{RESUMO}

Objetivos: Descrever um caso de Arterite de Takayasu diagnosticada durante o puerpério precoce, demonstrando a importância da aferição adequada da pressão arterial para o diagnóstico da doença hipertensiva gestacional.

Descrição do caso: Uma mulher de 40 anos, em sua quarta gestação, com idade gestacional de 36 semanas e três dias, foi hospitalizada por gestação de alto risco devido a hipertensão arterial sistêmica crônica. Durante a internação observou-se diferença nos níveis tensionais e assimetria de pulsos entre os membros superiores. No pós-parto a paciente foi submetida à ecografia de carótidas com Doppler, que demonstrou oclusão de artéria carótida comum esquerda e de artéria subclávia esquerda, levando ao diagnóstico de Arterite de Takayasu.

Conclusões: O diagnóstico precoce da Arterite de Takayasu é difícil, pois as manifestações iniciais são inespecíficas e os sintomas discretos. Entretanto, um exame físico cuidadoso pode evidenciar sinais que suscitem suspeitas e justifiquem investigação adicional, podendo prevenir um desfecho negativo, especialmente no período gestacional.

DESCRITORES: ARTERITE DE TAKAYASU; HIPERTENSÃO; HIPERTENSÃO INDUZIDA PELA GRAVIDEZ; CUIDADO PRÉ-NATAL; EXAME FÍSICO.

\section{ABSTRACT}

Aims: To describe a case of Takayasu arteritis diagnosed during the early postpartum period, demonstrating the importance of proper blood pressure measurement for the diagnosis of gestational hypertension.

Case description: A 40 year old woman in her fourth pregnancy, with gestational age of 36 weeks and three days, was hospitalized for highrisk pregnancy due to chronic hypertension. During hospitalization, difference in blood pressure levels and pulse asymmetry between the upper limbs were observed. In the postpartum the patient underwent carotid Doppler ultrasound, which showed occlusion of the left common carotid artery and left subclavian artery, leading to the diagnosis of Takayasu arteritis.

Conclusions: Early diagnosis of Takayasu arteritis is difficult because initial manifestations are nonspecific and symptoms are mild. However, a careful physical examination may reveal signs that raise suspicion and warrant further investigation, which may prevent a negative outcome, especially during pregnancy.

KEY WORDS: TAKAYASU ARTERITIS; HYPERTENSION; PREGNANCY INDUCED HYPERTENSION; PRENATAL CARE; PHYSICAL EXAMINATION.

Recebido em janeiro de 2014; aceito em maio de 2014 


\section{INTRODUÇÃO}

A hipertensão arterial sistêmica é definida por valores pressóricos sistólicos maiores ou iguais a $140 \mathrm{mmHg}$ e/ou diastólicos maiores ou iguais a $90 \mathrm{mmHg}$ em indivíduos adultos. ${ }^{1}$ Um exame físico detalhado é importante no diagnóstico da hipertensão arterial sistêmica, visto que são necessárias duas aferições da pressão arterial em pelo menos duas consultas médicas para se confirmar o diagnóstico, conforme o consenso da Sociedade Brasileira de Cardiologia. ${ }^{1,2}$ Uma adequada avaliação clínica pode estimar o risco cardiovascular e diagnosticar causas de hipertensão secundária, como doença de parênquima renal, estenose de artéria renal e outras anomalias vasculares, aldosteronismo primário, feocromocitoma e Síndrome de Cushing. Apesar de a hipertensão secundária ocorrer em apenas 3 a 5\% dos casos, o diagnóstico das suas causas é extremamente importante, visto que muitas delas são reversíveis. ${ }^{1}$

A Arterite de Takayasu é uma vasculite crônica de etiologia desconhecida que acomete preferencialmente a aorta e seus ramos primários. Essa alteração é decorrente de uma inflamação que leva ao afilamento dos vasos, podendo levar a estenoses, oclusões e formação de aneurismas. Dessa forma, esta patologia pode ser considerada uma importante causa de hipertensão secundária. A suspeita clínica ocorre quando se observa diferença de pressão arterial entre os membros superiores, por vezes associada à assimetria de pulsos. Segundo as diretrizes da European Society of Hypertension (ESH) e da European Society of Cardiology (ESC), ${ }^{1}$ recomenda-se um exame físico minucioso da pressão arterial, fazendo-se a aferição comparativa entre os membros superiores em pelo menos uma ocasião. Deve-se investigar possíveis anormalidades vasculares quando houver diferença entre as pressões arteriais sistólicas maior que $20 \mathrm{mmHg}$ e/ou entre as diastólicas maior que $10 \mathrm{mmHg}{ }^{1}$

A doença hipertensiva gestacional pode ser classificada em quatro subtipos: hipertensão gestacional, hipertensão crônica, pré-eclâmpsia e pré-eclâmpsia superposta à hipertensão crônica. O diagnóstico depende dos níveis de pressão arterial, valores de proteinúria e idade gestacional no momento do diagnóstico. Define-se a pré-eclâmpsia quando a paciente apresenta aumento de níveis de pressão arterial (maior ou igual a 140/90 $\mathrm{mmHg}$ ), acompanhado de proteinúria (maior ou igual a $300 \mathrm{mg} / 24$ horas), acima da vigésima semana de gestação. Quando esse quadro está associado à hipertensão crônica prévia à gestação ou anterior à vigésima semana, tem-se a pré-eclâmpsia sobreposta à hipertensão crônica. ${ }^{2,3}$
A doença hipertensiva gestacional é uma das principais causas de morbimortalidade materna e fetal no mundo. Até o momento, não há causa ou tratamento definitivo para esse distúrbio. No entanto, existem fatores de risco associados que podem se agravar durante a gestação, como o caso da hipertensão arterial crônica. Sabe-se que pacientes com distúrbios hipertensivos devem ser acompanhadas em pré-natal de alto risco, com cuidado especial para os níveis pressóricos. ${ }^{2,3}$

Dessa forma, patologias que podem estar associadas à hipertensão secundária merecem uma atenção redobrada em mulheres que desejam engravidar, visando um desfecho favorável para o binômio mãefilho. Este relato de caso apresenta uma situação clínica em que a Arterite de Takayasu foi diagnosticada no puerpério precoce, aborda as implicações clínicas da doença e, ainda, enfatiza a necessidade de um minucioso exame físico, principalmente em gestantes de alto risco. O relato foi aprovado pelo Comitê de Ética em Pesquisa da Pontifícia Universidade Católica do Rio Grande do Sul em dezembro de 2013, sob o parecer consubstanciado № 497.070.

\section{RELATO DO CASO}

Uma gestante de 40 anos de idade, casada, empregada doméstica, em sua quarta gestação, foi encaminhada do posto de saúde para acompanhamento no pré-natal de alto risco no Hospital São Lucas da PUCRS por hipertensão arterial crônica, diagnosticada aos 32 anos. Desde o diagnóstico da gestação a paciente vinha em uso de Metildopa $500 \mathrm{mg}, 1$ comprimido de $12 \mathrm{em}$ 12 horas. Quando estava com idade gestacional de 36 semanas e três dias, em uma consulta de pré-natal, a pressão arterial aferida foi de $190 \times 120 \mathrm{mmHg}$, confirmada por nova medida após um período de repouso de 10 minutos. Mediante esse aumento dos níveis pressóricos, a paciente foi internada no Hospital São Lucas da PUCRS. Durante a internação, a dosagem de proteinúria foi de $358 \mathrm{mg} / 24$ horas, o que confirmou o diagnóstico de pré-eclâmpsia sobreposta à hipertensão crônica. Outros exames de gravidade para avaliar possíveis complicações da pré-eclâmpsia, como a síndrome HELLP (hemolysis, elevated liver enzymes, low platelet count) também foram realizados, estando todos dentro do valor normal. Ainda na internação, foi observada diferença nos níveis pressóricos entre os membros superiores (membro superior esquerdo $110 \times$ $60 \mathrm{mmHg}$ e membro superior direito $170 \times 90 \mathrm{mmHg}$ ).

Durante quatro dias de internação, foi prescrita Nifedipina $10 \mathrm{mg}$, via oral, em caso de pressão arterial sistólica maior ou igual a $160 \mathrm{mmHg}$ e/ou diatólica maior 
ou igual a $110 \mathrm{mmHg}$, medicação que a paciente usou uma vez nos dois primeiros dias de internação, e em três momentos, no terceiro e quarto dia de internação. Tendo em vista a gravidade do caso, a equipe obstétrica optou pela indução do parto, inicialmente com misoprostol $25 \mu \mathrm{g}$, via vaginal. Em decorrência de colo desfavorável e dos níveis hipertensivos, foi indicado o parto cesáreo. O recém-nascido, do sexo masculino, pesou $2640 \mathrm{~g}$, sendo o percentil do peso em relação à idade gestacional abaixo de 10 (pequeno para a idade gestacional). O índice de Apgar foi 7 no primeiro minuto e 8 no quinto minuto. Durante a internação no alojamento conjunto obstétrico após o parto, evidenciaram-se novamente diferença nos níveis pressóricos entre os membros superiores da paciente (membro superior esquerdo: $110 \times$ $82 \mathrm{mmHg}$ e membro superior direito: $150 \times 88 \mathrm{mmHg}$ ). Identificou-se também assimetria de pulsos radiais, sendo solicitada uma ecografia com Doppler de artérias subclávias, carótidas e vertebrais. O exame demonstrou oclusão da artéria carótida comum esquerda (Figura 1A) e da artéria subclávia esquerda. A artéria carótida interna recebia fluxo por inversão da artéria carótida externa (Figura 1B) e a artéria axilar esquerda recebia fluxo por colateral da artéria torácica lateral. Tais achados levaram ao diagnóstico de Arterite de Takayasu. Foi também realizada uma angiorressonância de aorta e artérias renais, que não demonstrou alteração nesses segmentos vasculares. A paciente recebeu alta hospitalar com plano de acompanhamento no Ambulatório de Hipertensão Gestacional do Serviço de Nefrologia, em uso de captopril $50 \mathrm{mg}$ de $12 \mathrm{em} 12$ horas.

$\mathrm{Na}$ primeira consulta ambulatorial, duas semanas após a alta hospitalar, a paciente relatava parestesias em membro superior esquerdo, palpitações, cefaléia e eventual dispneia. Ao exame físico, apresentava pressão arterial em membro superior direito de $148 \times$ $110 \mathrm{mmHg}$ e no esquerdo não foi possível a aferição. O pulso carotídeo esquerdo estava diminuído e o pulso radial ipsilateral não era palpável. Apresentou melhora dos sintomas e controle da pressão arterial com o uso de anlodipina $5 \mathrm{mg}$ uma vez por dia, enalapril $20 \mathrm{mg}$ de 12 em 12 horas e hidroclorotiazida $25 \mathrm{mg}$ uma vez por dia. Com esse esquema terapêutico, os níveis pressóricos em membro superior direito foram a $137 \times 80 \mathrm{mmHg}$ e no membro superior esquerdo a $119 \times 82 \mathrm{mmHg}$.

Dois anos e meio após, a paciente apresentou piora da atividade da doença, com dor precordial, alterações visuais, cefaleia, parestesias e elevação de marcadores inflamatórios (velocidade de sedimentação globular $78 \mathrm{~mm} / \mathrm{h}$ e proteína C-reativa $1,8 \mathrm{mg} / \mathrm{dl}$ ). Nesse período, precisou ser internada para avaliação, sendo realizada nova ecografia com Doppler e arteriografia de membros superiores, que demonstraram as mesmas alterações prévias. No entanto, devido ao fato de a arteriografia evidenciar boa circulação colateral, não foi necessária intervenção cirúrgica. Os resultados da ecocardiografia e da cintilografia miocárdica com dipiridamol foram considerados normais. A paciente recebeu alta hospitalar após dezenove dias de internação, tendo iniciado com prednisona $10 \mathrm{mg}$ ao dia, metotrexato $2,5 \mathrm{mg} 8$ comprimidos uma vez por semana e cilostazol $100 \mathrm{mg}$ de $12 \mathrm{em} 12$ horas. Foi ajustado o esquema anti-hipertensivo para hidroclorotiazida $25 \mathrm{mg}$ uma vez ao dia, enalapril $10 \mathrm{mg}$ de $12 \mathrm{em} 12$ horas e anlodipina $10 \mathrm{mg}$ dois comprimidos ao dia e prescrito também ácido acetil salicílico $100 \mathrm{mg}$ uma vez ao dia. Com esse tratamento a paciente mantém-se assintomática, em acompanhamento ambulatorial há três anos.
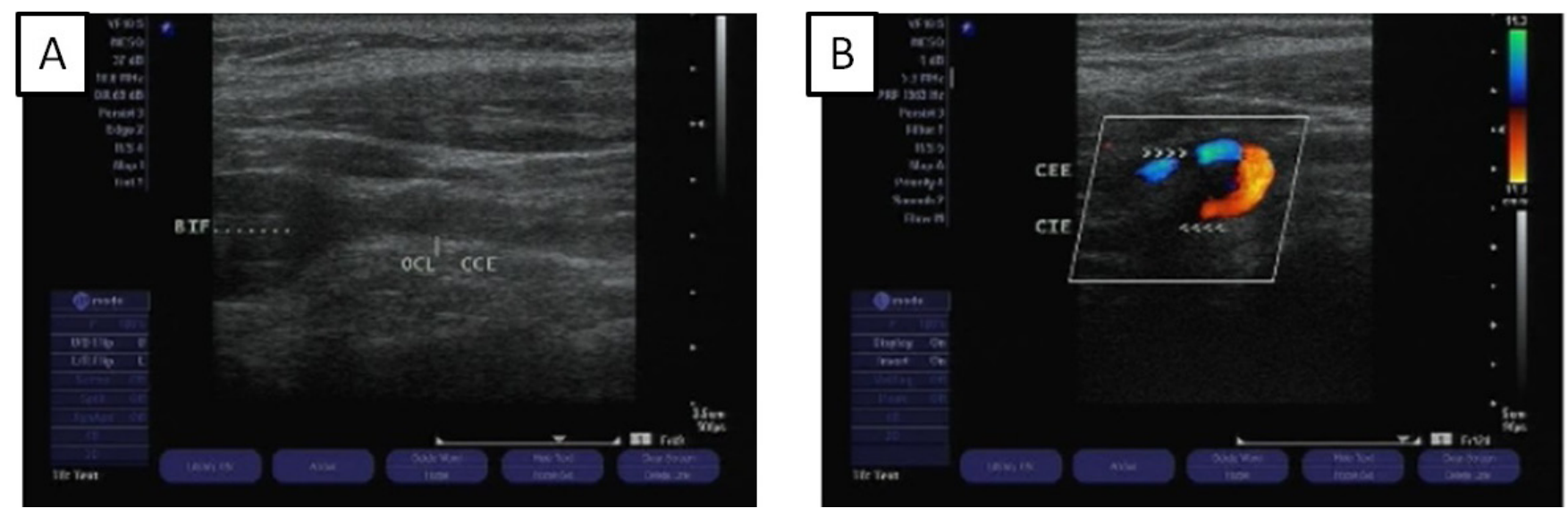

Figura 1. Ecodoppler colorido de artérias carótidas, em puérpera com pré-eclâmpsia sobreposta à hipertensão arterial sistêmica crônica, secundária a Arterite de Takayasu. (A) Oclusão de artéria carótida comum à esquerda. (B) Artéria carótida interna recebe fluxo por inversão da artéria carótida externa.

Veja esta figura em maior definição: http://revistaseletronicas.pucrs.br/ojs/index.php/scientiamedica/article/view/16332/11503 


\section{DISCUSSÃO}

A Arterite de Takayasu é uma vasculite crônica de etiologia desconhecida, sendo a grande maioria dos casos detectada em mulheres. A doença envolve artérias de grande e médio calibres, apresentando predileção pela aorta e seus ramos principais. O acometimento dos vasos é de inflamação transmural, podendo levar a estenose, oclusão e aneurismas. Para o diagnóstico de Arterite de Takayasu, o American College of Rheumatology utiliza os seguintes critérios: idade inferior a 40 anos, claudicação de extremidades, diminuição de pulsos braquiais, diferença sistólica entre membros superiores acima de $10 \mathrm{mmHg}$, sopro sobre artérias subclávias ou aorta e alterações arteriográficas, sendo necessária a presença de pelo menos três critérios. ${ }^{4} \mathrm{O}$ tratamento tem como base os corticosteroides, que produzem resposta clínica em torno de $50 \%$ dos casos. Outros imunossupressores, tais como metotrexato, podem ser utilizados. O controle da pressão arterial é extremamente importante para o manejo clínico. Deve-se ainda recomendar atenção para o risco de trombose nesses pacientes. ${ }^{5}$ A paciente do caso relatado sabia-se hipertensa desde os seus 32 anos de idade. É fundamental destacar que a alta suspeita através da história clínica e a atenção no exame físico e na aferição da pressão arterial são imprescindíveis para a detecção da doença. É a suspeita clínica que guiará a investigação complementar, podendo levar ao diagnóstico oportuno e, no caso de uma gestante, podendo minimizar os desfechos materno-fetais desfavoráveis, já que o tratamento é capaz de impedir a progressão da doença em um grande número de pacientes. $^{8}$

Estudos prévios que abordaram Arterite de Takayasu na gestação deixam bem clara a importância do acompanhamento multidisciplinar, por obstetra, reumatologista e cardiologista, tanto no período prénatal quanto a longo prazo. Sabe-se que um bom prénatal promove um melhor desfecho perinatal nessas pacientes, que são mais propensas a desenvolver hipertensão, pré-eclâmpsia e crescimento intra-uterino restrito, complicações vistas no presente caso. ${ }^{6-9}$

Este relato de caso salienta o quanto um exame físico cuidadoso pode influenciar no diagnóstico e evolução da Arterite de Takayasu, patologia que está associada à principal causa de morte materna no Brasil, que é a doença hipertensiva gestacional. A arterite de Takayasu apresenta características que podem ser detectadas, porém somente a alta suspeitabilidade e a atenção ao exame clínico irão prover as ferramentas para o seu diagnóstico. Na evidência da redução na amplitude de um ou mais pulsos, diferenças na pressão arterial entre os membros, ou sopros na região cervical, área supraclavicular, axilar ou abdominal, é possível fazer o diagnóstico desta doença que, se adequadamente diagnosticada e tratada, permite um desfecho favorável para o binômio mãe-filho.

\section{REFERÊNCIAS}

1. Mancia G, Fagard R, Narkiewicz K, Redon J, Zanchetti A, Böhm M, Christiaens T, Cifkova R, De Backer G, Dominiczak A, Galderisi M, Grobbee DE, Jaarsma T, Kirchhof P, Kjeldsen SE, Laurent S, Manolis AJ, Nilsson PM, Ruilope LM, Schmieder RE, Sirnes PA, Sleight P, Viigimaa M, Waeber B, Zannad F; ESH Scientific Council, Redon J, Dominiczak A, Narkiewicz K, Nilsson PM, Burnier M, Viigimaa M, Ambrosioni E, Caufield M, Coca A, Olsen MH, Schmieder RE, Tsioufis C, van de Borne P; ESC Committee for Practice Guidelines (CPG), Zamorano JL, Achenbach S, Baumgartner H, Bax JJ, Bueno H, Dean V, Deaton C, Erol C, Fagard R, Ferrari R, Hasdai D, Hoes AW, Kirchhof P, Knuuti J, Kolh P, Lancellotti P, Linhart A, Nihoyannopoulos P, Piepoli MF, Ponikowski P, Sirnes PA, Tamargo JL, Tendera M, Torbicki A, Wijns W, Windecker S; Document Reviewers, Clement DL, Coca A, Gillebert TC, Tendera M, Rosei EA, Ambrosioni E, Anker SD, Bauersachs J, Hitij JB, Caulfield M, De Buyzere M, De Geest S, Derumeaux GA, Erdine S, Farsang C, FunckBrentano C, Gerc V, Germano G, Gielen S, Haller H, Hoes AW, Jordan J, Kahan T, Komajda M, Lovic D, Mahrholdt H, Olsen MH, Ostergren J, Parati G, Perk J, Polonia J, Popescu BA, Reiner Z, Rydén L, Sirenko Y, Stanton A, Struijker-Boudier H, Tsioufis C, van de Borne P, Vlachopoulos C, Volpe M, Wood DA. ESH/ESC Guidelines for the management of arterial hypertension: The Task Force for the management of arterial hypertension of the European Society of Hypertension (ESH) and of the European Society of Cardiology (ESC). Eur Heart J. 2013;34(28):2159-219.

2. VI Brazilian Guidelines on Hypertension. Arq Bras Cardiol. 2010;95(1 Suppl):1-51.

3. Steegers EA, von Dadelszen P, Duvekot JJ, Pijnenborg R. Pre-eclampsia. Lancet. 2010;376(9741):631-44.

4. Fauci AS, Leavitt RY, Lie JT, Lightfoot Jr RW, Masi AT, McShane DJ, Mills JA, Stevens MB, Wallace SL, Zvaifler NJ. The American College of Rheumatology 1990 criteria for the classification of Takayasu arteritis. Arthritis Rheum. 1990;33(8):1129-34.

5. Kerr GS, Hallahan CW, Giordano J, Leavitt RY, Fauci AS, Rottem M, Hoffman GS. Takayasu arteritis. Ann Intern Med. 1994;1120(11):919-29.

6. de Jesus GR, d'Oliveira IC, dos Santos FC, Rodrigues G, Klumb EM, de Jesus NR,Levy RA. Pregnancy may aggravate arterial hypertension in women with Takayasu arteritis. Isr Med Assoc J. 2012;14(12):724-8.

7. Li LT, Gilani R, Tsai PI, Wall MJ, Jr. Takayasu arteritis complicating pregnancy in adolescence. Ann Vasc Surg. 2012;26(6):858 e7-10.

8. Shafi NA, Malik A, Silverman DI. Management of Takayasu arteritis during pregnancy. J Clin Hypertens (Greenwich). 2009;11(7):383-5.

9. Papantoniou N, Katsoulis I, Papageorgiou I, Antsaklis A. Takayasu arteritis in pregnancy:safe management options in antenatal care. Case report. Fetal Diagn Ther. 2007;22(6):449-51. 